Pacific Journal of Mathematics

THE MODULUS OF A DOUBLY CONNECTED REGION AND
THE GEODESIC CURVATURE-AREA METHOD 


\title{
THE MODULUS OF A DOUBLY CONNECTED REGION AND THE GEODESIC CURVATURE-AREA METHOD
}

\section{DAVID Minda}

\begin{abstract}
It is well known that the modulus of a doubly connected Riemann surface can be determined by the length-area method, that is, the method of extremal length, and that the extremal metric can be expressed in terms of a quadratic differential. Ahlfors introduced a related method based on the comparison of geodesic curvature and area. We show that the modulus of a doubly connected Riemann surface can be obtained by means of this geodesic curvature-area method. In the important special case in which there is a restriction on the curvature of the metrics, we identify all extremal metrics; they have constant curvature.
\end{abstract}

1. Introduction. A comparison of length and area has led to many important results in complex analysis. This method is based upon the fact that length and area are invariant under a conformal mapping when the metric undergoes a corresponding transformation. Ahlfors [2] considered a third conformally invariant quantity: geodesic curvature. He initiated a program based on the comparison of geodesic curvature and area. $\mathrm{He}$ presented the basic principles of the method and applied it to one simple case - the problem of estimating the conformal radius of a simply connected region. This is equivalent to estimating the hyperbolic metric on a simply connected region. He obtained explicit, sharp upper and lower bounds. The method is limited to smooth metrics. By making use of other methods, Minda [5] extended the upper bound to the class of $S K(k)$ metrics.

The work of Ahlfors indicates that the method has a wider range of applicability. But it is not clear that it will lead to equally explicit results in other situations. We apply the geodesic curvature-area method to the next simplest problem - estimation of the modulus of a doubly connected surface - and obtain an explicit, sharp upper bound.

2. Conformal metrics. In this section we gather together some basic facts concerning conformal metrics on Riemann surfaces. We adopt the convention, to be in effect for the remainder of the paper, that all metrics are positive and of class $C^{2}$. Let $X$ be a Riemann surface and $\rho(z)|d z|$ a 
metric on $X$. The (Gaussian) curvature of this metric is defined to be

$$
\kappa(z, \rho)=-\frac{\Delta \log \rho(z)}{\rho^{2}(z)} .
$$

Let $\mathbf{D}=\{z:|z|<1\}$. The Poincaré hyperbolic metric on $\mathbf{D}$ is

$$
\lambda_{\mathbf{D}}(z)|d z|=\frac{2|d z|}{1-|z|^{2}}
$$

it has constant curvature -1 .

The pull-back of a metric via an analytic mapping is an important concept. Suppose $f: Y \rightarrow X$ is a locally schlicht analytic function and $\rho(z)|d z|$ is a metric on $X$. The pull-back to $Y$ via $f$ of $\rho(z)|d z|$ is a metric on $Y$ denoted by $f^{*}(\rho(z)|d z|)$. In case $X$ and $Y$ are plane regions, $f^{*}(\rho(z)|d z|)=\rho(f(z))\left|f^{\prime}(z)\right||d z|$. For Riemann surfaces an analogous formula holds, but it is necessary to work with local coordinates. It is elementary to verify that

$$
\kappa(f(z), \rho)=\kappa\left(z, f^{*}(\rho)\right) .
$$

In particular, curvature is a conformal invariant. If $\gamma$ is a path on $Y$, then

$$
\int_{\gamma} f^{*}(\rho(z)|d z|)=\int_{f \circ \gamma} \rho(z)|d z| .
$$

Thus, length is a conformal invariant. Now, set $\sigma(z)|d z|=f^{*}(\rho(z)|d z|)$. If $\Omega$ is a Borel measurable subset of $Y$, then

$$
\iint_{\Omega} \sigma^{2}(z) d x d y=\iint_{f(\Omega)} \rho^{2}(z) d x d y
$$

provided $f$ is injective. Hence, area is a conformal invariant.

We are interested in another geometric quantity which is also a conformal invariant. Let $\gamma:[a, b] \rightarrow X$ be an arc on $X$. The total geodesic curvature of the oriented arc relative to the metric $\rho(z)|d z|$ can be computed from the formula

$$
\begin{aligned}
G_{\rho}(\gamma) & =\int_{\gamma} d(\arg d z)+\int_{\gamma} * d \log \rho(z) \\
& =\int_{\gamma} d(\arg d z)+\int_{\gamma} \frac{\partial \log \rho(z)}{\partial n} d s,
\end{aligned}
$$

where the normal derivative is taken in the direction of the right-hand normal. Direct calculation gives

$$
G_{\rho}(f \circ \gamma)=G_{f^{*}(\rho)}(\gamma)
$$


if $f: Y \rightarrow X$ is a conformal mapping and $\gamma$ is a path on $Y$. A few examples might be helpful. If $\gamma$ is a subarc of a hyperbolic line in $\mathbf{D}$, then $G_{\lambda_{\mathbf{D}}}(\gamma)=0$. If $\lambda_{\mathbf{C}}(z)|d z|=|d z|$ is the euclidean metric on $\mathbf{C}$ and $\gamma$ is a straight line segment, then $G_{\lambda_{c}}(\gamma)=0$. The Gauss-Bonnet Theorem relates the curvature and total geodesic curvature. Suppose $\rho(z)|d z|$ is a metric on $X$. Let $\Omega$ be a regularly embedded subregion and $\partial \Omega$ the positively oriented boundary of $\Omega$. Then

$$
-\iint_{\Omega} \kappa(z, \rho) \rho^{2}(z) d x d y=G_{\rho}(\partial \Omega)-\int_{\partial \Omega} d(\arg d z) .
$$

Finally, we introduce the hyperbolic metric. Let $X$ be a hyperbolic Riemann surface; that is, the unit disk $\mathbf{D}$ is the universal covering surface of $X$. The hyperbolic metric $\lambda_{X}(z)|d z|$ on $X$ is the unique metric with the following property: if $\pi: \mathbf{D} \rightarrow X$ is any analytic universal covering projection, then $\pi^{*}\left(\lambda_{X}(z)|d z|\right)=\lambda_{\mathbf{D}}(z)|d z|$. Thus, $\lambda_{X}(z)|d z|$ has constant curvature -1 . Let $\Omega(r)=\{z: 1 / r<|z|<r\}$, where $1<r<\infty$. The hyperbolic metric on $\Omega(r)$ is

$$
\lambda_{r}(z)|d z|=\frac{\pi}{2 \log r} \frac{|d z|}{|z| \cos \left(\left(\frac{1}{2} \pi \log |z|\right) / \log r\right)} .
$$

3. Standard metric for a doubly connected surface. Let $X$ be a proper doubly connected Riemann surface. That is, $X$ is conformally equivalent to $\Omega(r)$ for some $r \in(1, \infty)$. The modulus of $X$ is defined to be $\bmod (X)=(1 / 2 \pi) \log r^{2}$ and it is a conformal invariant. We shall specify a standard metric of constant curvature 0 on $X$. Let $\gamma_{0}$ be any generator of the fundamental group of $X$ and $\mathcal{H}$ the family of all closed curves $\gamma$ on $X$ which are freely homotopic to $\gamma_{0}$. Fix a positive number $a$. A metric $\sigma(z)|d z|$ on $X$ is called admissible if

$$
\int_{\gamma} \sigma(z)|d z| \geq a
$$

for all locally rectifiable $\gamma \in \mathscr{H}$. This is equivalent to asserting that (1) holds for any locally rectifiable path $\gamma$ which generates the fundamental group of $X$. Let

$$
A_{\sigma}(X)=\iint_{X} \sigma^{2}(z) d x d y
$$

denote the area of $X$ relative to the metric $\sigma(z)|d z|$ and

$$
M(a)=\inf A_{\sigma}(X),
$$


where the infimum is taken over all admissible metrics. A very special case of a result of Jenkins [4] implies that there is a unique real-analytic extremal metric $\sigma_{a}(z)|d z|$ for this problem. It has the form $\left|Q_{a}(z)\right|^{1 / 2}|d z|$, where $Q_{a}(z) d z^{2}$ is a holomorphic quadratic differential on $X$. Moreover, $A_{\sigma_{a}}(X) \leq A_{\sigma}(X)$ for any admissible metric $\sigma(z)|d z|$ with strict inequality unless $\sigma(z)|d z|=\sigma_{a}(z)|d z|$. It is straightforward to verify that $\sigma_{a}(z)|d z|=a \sigma_{1}(z)|d z|$. We define $\sigma_{X}(z)|d z|=\sigma_{1}(z)|d z|$. Since $\sigma_{X}(z)|d z|=\left|Q_{1}(z)\right|^{1 / 2}|d z|$, where $Q_{1}(z) d z^{2}$ is a holomorphic quadratic differential, the metric $\sigma_{X}(z)|d z|$ is locally euclidean and has constant curvature zero. It is not difficult to show that $\sigma_{X}(z)|d z|$ is independent of the choice of generator of the fundamental group of $X$. Also, this standard metric is a conformal invariant; that is, if $f: X \rightarrow Y$ is a conformal mapping of proper doubly connected surfaces, then $f^{*}\left(\sigma_{Y}(z)|d z|\right)=$ $\sigma_{X}(z)|d z|$. In particular, $\sigma_{X}(z)|d z|$ is invariant under the group $\operatorname{Aut}(X)$ of conformal automorphisms of $X$.

It is known that

$$
\sigma_{\Omega(r)}(z)|d z|=\frac{1}{2 \pi} \frac{|d z|}{|z|} ;
$$

recall that $|d z| /|z|$ is the logarithmic metric on the punctured plane $\mathrm{C} \backslash\{0\}$. Then

$$
A_{\sigma_{\Omega(r)}}(\Omega(r))=\iint_{\Omega(r)} \sigma_{\Omega(r)}^{2} d x d y=\frac{1}{2 \pi} \log r^{2}=\bmod \Omega(r) .
$$

Now, assume that $X$ is a proper doubly connected surface and $f: X \rightarrow \Omega(r)$ is a conformal mapping. Then $\sigma_{X}(z)|d z|=f^{*}\left(\sigma_{\Omega(r)}(z)|d z|\right)$. Due to the conformal invariance of the quantities involved, we conclude that $\bmod (X)$ $=A_{\sigma_{X}}(X)$ from (2). Thus, $\bmod (X) \leq A_{\sigma}(X)$ for any metric $\sigma(z)|d z|$ admissible for $a=1$ with equality only if $\sigma(z)|d z|=\sigma_{X}(z)|d z|$.

There is a simple relationship between $\sigma_{X}(z)|d z|$ and the hyperbolic metric.

LEMMA. Let $X$ be a proper doubly connected Riemann surface and $\lambda_{X}(z)|d z|$ the hyperbolic metric on $X$. Then

$$
\sigma_{X}(z)|d z| \leq \frac{\bmod (X)}{\pi} \lambda_{X}(z)|d z|
$$

and this inequality is sharp.

Proof. Because of the conformally invariant character of the quantities involved in inequality (3), it is sufficient to establish (3) in the special 
case $X=\Omega(r)$. Now,

$$
\begin{aligned}
\lambda_{\Omega(r)}(z)|d z| & =\frac{\pi}{2 \log r} \frac{|d z|}{|z| \cos \left(\left(\frac{1}{2} \pi \log |z|\right) / \log r\right)} \\
& \geq \frac{\pi}{2 \log r} \frac{|d z|}{|z|}=\frac{\pi}{\bmod \Omega(r)} \sigma_{\Omega(r)}(z)|d z|,
\end{aligned}
$$

which establishes (3) in this special case. Note that equality holds if and only if $|z|=1$.

4. Main result. First, we introduce some terminology and notation. Let $X$ be a doubly connected Riemann surface. A regularly embedded doubly connected region $\Omega \subset X$ is called admissible if it separates the two boundary components of $X$. If $\rho(z)|d z|$ is a metric on $X$ and $\Omega$ is an admissible region, then the total geodesic curvature of $\partial \Omega$ with respect to $\rho(z)|d z|$ is

$$
G_{\rho}(\partial \Omega)=\int_{\partial \Omega} * d \log \rho(z)
$$

since $\int_{\partial \Omega} d(\arg d z)=0$. A closed path $\gamma$ on $X$ is admissible if it is a generator of the fundamental group of $X$. Given a positive number $a$, we say that a metric $\rho(z)|d z|$ on $X$ is admissible if

$$
\log a \leq \int_{\gamma} \log \left[\frac{\rho(z)}{\sigma_{X}(z)}\right] \sigma_{X}(z)|d z|
$$

for all admissible paths $\gamma$. In this case we write $\rho(z)|d z| \in \Gamma(a)$. We note that this condition is invariant under conformal mappings. Roughly speaking, this condition asserts that $\rho(z)|d z|$ is not too small compared to $\sigma_{X}(z)|d z|$.

THEOREM 1. Let $X$ be a proper doubly connected Riemann surface. Suppose $\Phi:[0, \infty) \rightarrow[0, \infty)$ is either strictly increasing and $\Phi(0)=0$ or else $\Phi=0$. Set $\Lambda(x)=\frac{1}{2} \int_{0}^{x} \Phi(t) d t$ and $\Psi(x)=\int_{0}^{x} d t /\left(\Lambda(t)+a^{2}\right)$. If $\rho(z)|d z| \in \Gamma(a)$ and $G_{\rho}(\partial \Omega) \geq \Phi\left(A_{\rho}(\Omega)\right)$ for all admissible regions $\Omega$, then $\bmod (X) \leq \Psi\left(A_{\rho}(X)\right)$.

Proof. In view of the conformal invariance of the quantities involved, we may assume from the outset that $X=\Omega(r)$ for some $r \in(1, \infty)$. The condition that $\rho(z)|d z| \in \Gamma(a)$ becomes

$$
\log a \leq \int_{\gamma} \log (2 \pi|z| \rho(z)) \frac{|d z|}{2 \pi|z|}
$$


for all admissible paths $\gamma$. We slit $\Omega(r)$ along the negative real axis to obtain a simply connected region $\tilde{\Omega}(r)$. Then $z(w)=\exp (w)$ is a conformal mapping of the rectangle $Q(r)=\{w=u+i v:|u|<\log r,|v|<\pi\}$ onto $\tilde{\Omega}(r)$. Let $\sigma(w)|d w|=\exp ^{*}(\rho(z)|d z|)$. Explicitly, $\rho(z(w))=e^{-u} \sigma(w)$. The path $\gamma(v)=\exp (i v),-\pi \leq v \leq \pi$, is admissible and inequality (4) becomes

$$
\begin{aligned}
\log a & \leq \int_{-\pi}^{\pi} \log \left(2 \pi \rho\left(e^{i v}\right)\right) \frac{d v}{2 \pi} \\
& =\frac{1}{2 \pi} \int_{-\pi}^{\pi} \log \sigma(0, v) d v+\log (2 \pi) .
\end{aligned}
$$

For $t \in[0, \log r)$ the region $\Omega\left(e^{t}\right)$ is admissible. The area of $\Omega\left(e^{t}\right)$ is

$$
A(t)=\iint_{\Omega\left(e^{t}\right)} \rho^{2}(z) d x d y=\int_{-t}^{t} \int_{-\pi}^{\pi} \sigma^{2}(u, v) d u d v .
$$

Also, the total geodesic curvature of the positively oriented boundary $\partial \Omega\left(e^{t}\right)$ is

$$
\begin{aligned}
G(t) & =G_{\rho}\left(\partial \Omega\left(e^{t}\right)\right)=\int_{\partial \Omega\left(e^{t}\right)} * d \log \rho(z) \\
& =\int_{-\pi}^{\pi} \frac{\partial}{\partial t} \log \sigma(t, v) d v-\int_{-\pi}^{\pi} \frac{\partial}{\partial t} \log \sigma(-t, v) d v .
\end{aligned}
$$

Therefore,

$$
\begin{aligned}
\int_{0}^{u} G(t) d t= & \int_{-\pi}^{\pi} \log \sigma(u, v) d v \\
& +\int_{-\pi}^{\pi} \log \sigma(-u, v) d v-2 \int_{-\pi}^{\pi} \log \sigma(0, v) d v
\end{aligned}
$$

or

$$
\begin{aligned}
\frac{1}{2 \pi} \int_{0}^{u} G(t) d t & +\frac{1}{\pi} \int_{-\pi}^{\pi} \log \sigma(0, v) d v \\
& =\frac{1}{2 \pi} \int_{-\pi}^{\pi} \log (\sigma(u, v) \sigma(-u, v)) d v \\
& \leq \log \frac{1}{2 \pi} \int_{-\pi}^{\pi} \sigma(u, v) \sigma(-u, v) d v \\
& \leq \log \frac{1}{4 \pi} \int_{-\pi}^{\pi}\left(\sigma^{2}(u, v)+\sigma^{2}(-u, v)\right) d v
\end{aligned}
$$


This gives

$$
\begin{aligned}
& \exp \left(\frac{1}{2 \pi} \int_{0}^{u} G(t) d t+\frac{1}{\pi} \int_{-\pi}^{\pi} \log \sigma(0, v) d v\right) \\
& \quad \leq \frac{1}{4 \pi} \int_{-\pi}^{\pi}\left(\sigma^{2}(u, v)+\sigma^{2}(-u, v)\right) d v=\frac{1}{4 \pi} A^{\prime}(u) .
\end{aligned}
$$

By making use of the assumption $G(t) \geq \Phi(A(t))$, we obtain

$$
\begin{aligned}
A^{\prime}(u) & \geq \exp \left(\frac{1}{2 \pi} \int_{0}^{u} \Phi(A(t)) d t+\frac{1}{\pi} \int_{-\pi}^{\pi} \log \sigma(0, v) d v+\log (4 \pi)\right) \\
& =\exp B(u),
\end{aligned}
$$

where $B(u)$ is an abbreviation for the preceding expression in parentheses. Observe that

$$
B(0)=\frac{1}{\pi} \int_{-\pi}^{\pi} \log \sigma(0, v)+\log (4 \pi) \geq \log \left(\frac{a^{2}}{\pi}\right)
$$

by inequality (5).

We now treat the case $\Phi=0$. In this situation $\Lambda=0$ and $\Psi(x)=$ $x / a^{2}$. Also, $B(u)=B(0)$ for all $u$ since $\Phi=0$. Thus,

$$
\frac{a^{2}}{\pi} \leq \exp B(0) \leq A^{\prime}(t), \quad \frac{u}{\pi} \leq \int_{0}^{u} \frac{A^{\prime}(t)}{a^{2}} d t=\Psi(A(u)) .
$$

The choice $u=\log r$ gives

$$
\bmod \Omega(r)=\frac{\log r}{\pi} \leq \Psi(A(\log r))=\Psi\left(A_{\rho}(\Omega(r))\right) .
$$

This establishes Theorem 1 in case $\Phi=0$.

Next, we consider the case in which $\Phi$ is strictly increasing and $\Phi(0)=0$. The definition of $B$ gives

$$
B^{\prime}(t)=\frac{1}{2 \pi} \Phi(A(t))=\frac{1}{\pi} \Lambda^{\prime}(A(t)) .
$$

Thus,

$$
\frac{1}{\pi} \Lambda^{\prime}(A(t)) A^{\prime}(t) \geq \exp (B(t)) B^{\prime}(t)
$$

and integration over the interval $[0, u]$ results in

$$
\frac{1}{\pi} \Lambda(A(u)) \geq \exp B(u)-\exp B(0),
$$

or

$$
\log \left(\frac{1}{\pi} \Lambda(A(u))+\exp B(0)\right) \geq B(u) .
$$


Now, integrate the identity $1 / \pi=B^{\prime}(t) / \Lambda^{\prime}(A(t))$ over the interval $[\varepsilon, u]$, where $0<\varepsilon<u$.

$$
\begin{aligned}
\frac{1}{\pi}(u-\varepsilon) \leq & \int_{\varepsilon}^{u} \frac{B^{\prime}(t)}{\Lambda^{\prime}(A(t))} d t \\
= & \frac{B(u)}{\Lambda^{\prime}(A(u))}-\frac{B(\varepsilon)}{\Lambda^{\prime}(A(\varepsilon))}+\int_{\varepsilon}^{u} \frac{B(t) \Lambda^{\prime \prime}(A(t)) A^{\prime}(t)}{\Lambda^{\prime}(A(t))^{2}} d t \\
\leq & \frac{B(u)}{\Lambda^{\prime}(A(u))}-\frac{B(\varepsilon)}{\Lambda^{\prime}(A(\varepsilon))} \\
& +\int_{\varepsilon}^{u} \frac{\log \left(\Lambda(A(t)) / \pi+e^{B(0)}\right) \Lambda^{\prime \prime}(A(t)) A^{\prime}(t)}{\Lambda^{\prime}(A(t))^{2}} d t \\
= & \frac{B(u)-\log \left(\Lambda(A(u)) / \pi+e^{B(0)}\right)}{\Lambda^{\prime}(A(u))} \\
& +\frac{\log \left(\Lambda(A(\varepsilon)) / \pi+e^{B(0)}\right)-B(\varepsilon)}{\Lambda^{\prime}(A(\varepsilon))} \\
& +\int_{\varepsilon}^{u} \frac{A^{\prime}(t) / \pi}{\Lambda(A(u)) / \pi+e^{B(0)}} d t .
\end{aligned}
$$

By making use of inequalities (6) and (7) we find that

$$
\begin{aligned}
\frac{1}{\pi}(u-\varepsilon) & \leq \int_{\varepsilon}^{u} \frac{A^{\prime}(t)}{\Lambda(A(u))+a^{2}} d t+\frac{\log \left(\Lambda(A(\varepsilon)) / \pi+e^{B(0)}\right)-B(\varepsilon)}{\Lambda^{\prime}(A(\varepsilon))} \\
& \leq \Psi(A(u))+\frac{\log \left(\Lambda(A(\varepsilon)) / \pi+e^{B(0)}\right)-B(\varepsilon)}{\Lambda^{\prime}(A(\varepsilon))} .
\end{aligned}
$$

Assume that

$$
\lim _{\varepsilon \rightarrow 0} \frac{\log \left(\Lambda(A(\varepsilon)) / \pi+e^{B(0)}\right)-B(\varepsilon)}{\Lambda^{\prime}(A(\varepsilon))}=0 .
$$

Then we obtain

$$
\frac{u}{\pi} \leq \Psi(A(u))
$$

and the choice $u=\log r$ establishes Theorem 1 in this case.

In order to complete the proof of Theorem 1 we demonstrate (8). For $x$ and $y$ positive we have the elementary inequality

$$
\log (x+y) \leq \log (x)+(y / x) \text {. }
$$


Thus

$$
\begin{aligned}
0 & \leq \log \left(\frac{1}{\pi} \Lambda(A(\varepsilon))+e^{B(0)}\right)-B(\varepsilon) \\
& \leq B(0)-B(\varepsilon)+e^{-B(0)}\left(\frac{1}{\pi}\right) \Lambda(A(\varepsilon)) \\
& =\frac{1}{2 \pi} e^{-B(0)} \int_{0}^{A(\varepsilon)} \Phi(t) d t-\frac{1}{2 \pi} \int_{0}^{\varepsilon} \Phi(A(t)) d t \\
& =\frac{1}{2 \pi} \int_{0}^{\varepsilon} \Phi(A(t))\left[e^{-B(0)} A^{\prime}(t)-1\right] d t .
\end{aligned}
$$

Since $A^{\prime}(t) \geq e^{B(t)} \geq e^{B(0)}$, the second factor in the integrand is nonnegative. The generalized mean value theorem for integrals yields $\eta \in(0, \varepsilon)$ such that

$$
\begin{aligned}
\int_{0}^{\varepsilon} \Phi(A(t))\left[e^{-B(0)} A^{\prime}(t)-1\right] d t & =\left[e^{-B(0)} A^{\prime}(\eta)-1\right] \int_{0}^{\varepsilon} \Phi(A(t)) d t \\
& \leq \varepsilon\left[e^{-B(0)} A^{\prime}(\eta)-1\right] \Phi(A(\varepsilon)) .
\end{aligned}
$$

Because $\Lambda^{\prime}(A(\varepsilon))=\frac{1}{2} \Phi(A(\varepsilon))$, the limit (8) follows easily from the preceding work.

5. Sharpness. We now demonstrate that the upper bound given in Theorem 1 is sharp in the important case when there is a restriction on the curvature of the metric. We begin by reformulating Theorem 1 in this situation. Suppose $\rho(z)|d z|$ is a metric on $X$ and $\kappa(z, \rho) \leq-k$, where $k \geq 0$. If $\Omega$ is any admissible doubly connected subregion, then the Gauss-Bonnet Theorem gives

$$
G_{\rho}(\partial \Omega)=\iint_{\Omega}-\kappa(z, \rho) \rho^{2}(z) d x d y \geq k \iint_{\Omega} \rho^{2}(z) d x d y=k A_{\rho}(\Omega) .
$$

Thus, if $\Phi_{k}(x)=k x$, then $G_{\rho}(\partial \Omega) \geq \Phi_{k}\left(A_{\rho}(\Omega)\right)$ for any admissible region $\Omega$. Set

$$
\Lambda_{k}(x)=\frac{1}{2} \int_{0}^{x} \Phi_{k}(t) d t=k \frac{x^{2}}{4}
$$

and

$$
\Psi_{k}(x)=\int_{0}^{x} \frac{d t}{\Lambda_{k}(t)+a^{2}}= \begin{cases}\frac{x}{a^{2}} & \text { if } k=0, \\ \frac{2}{\sqrt{k} a} \arctan \left(\frac{\sqrt{k} x}{2 a}\right) & \text { if } k>0 .\end{cases}
$$


Observe that $\Psi_{k}(x)<\Psi_{0}(x)$ for $x>0$ and $k>0$ and that $\Psi_{k} \rightarrow \Psi_{0}$ pointwise as $k \rightarrow 0$. We restate Theorem 1 in this context.

THEOREM 2. Let $X$ be a proper doubly connected Riemann surface. If $\rho(z)|d z| \in \Gamma(a)$ and $\kappa(z, \rho) \leq-k$, where $k \geq 0$, then $\bmod (X) \leq$ $\Psi_{k}\left(A_{\rho}(X)\right)$.

We shall demonstrate that this upper bound is sharp for any $a>0$ and $k \geq 0$. It is sufficient to consider the special case $X=\Omega(r)$ due to the conformally invariant nature of the theorem. First, we treat the case in which $k=0$. For any $a>0$ define $\rho_{a}(z)|d z|=a \sigma_{\Omega(r)}(z)|d z|$. Then $\rho_{a}(z)|d z|$ has curvature zero and belongs to $\Gamma(a)$. Also,

$$
A_{\rho_{a}}(\Omega(r))=a^{2} A_{\sigma_{\Omega(r)}}(\Omega(r))=a^{2} \bmod \Omega(r),
$$

or

$$
\bmod \Omega(r)=A_{\rho_{a}}(\Omega(r)) / a^{2}=\Psi_{0}\left(A_{\rho_{a}}(\Omega(r))\right) .
$$

Thus, $\rho_{a}(z)|d z|$ is an extremal metric for $k=0$.

Now, we turn to the case in which $k>0$. We begin by demonstrating that if $\rho(z)|d z| \in \Gamma(a)$ and $\kappa(z, \rho) \leq-k$, then

$$
a \leq \frac{\pi}{\sqrt{k} \bmod (\Omega(r))} .
$$

Let $\gamma$ denote the positively oriented unit circle. Then for $z \in \gamma$

$$
\frac{\rho(z)}{\sigma_{\Omega(r)}(z)}=\frac{\rho(z)}{\lambda_{\Omega(r)}(z)} \frac{\pi}{\bmod (\Omega(r))} .
$$

Now, $\sqrt{k} \rho$ has curvature at most -1 , so Ahlfors' extension of Schwarz' lemma [1] implies that $\sqrt{k} \rho \leq \lambda_{\Omega(r)}$. Therefore, for $z \in \gamma$,

$$
\frac{\rho(z)}{\sigma_{\Omega(r)}(z)} \leq \frac{\pi}{\sqrt{k} \bmod (\Omega(r))} .
$$

Since $\gamma$ has length 1 relative to $\sigma_{\Omega(r)}(z)|d z|$ and $\rho(z)|d z| \in \Gamma(a)$, we obtain the desired result.

Fix $k>0$. Then inequality (9) implies that we may select $R \in[r, \infty)$ so that

$$
a=\frac{\pi}{\sqrt{k} \bmod \Omega(R)}=\frac{\pi^{2}}{\sqrt{k} \log R} .
$$


Now, $\rho_{a}(z)|d z|=(1 / \sqrt{k}) \lambda_{R}(z)|d z|$ is defined on $\Omega(r)$ and has constant curvature $-k$. Also,

$$
\begin{aligned}
\frac{\rho_{a}(z)}{\sigma_{\Omega(r)}(z)} & =\frac{\pi^{2}}{\sqrt{k} \log R} \cdot \frac{1}{\cos \left(\left(\frac{1}{2} \pi \log |z|\right) / \log R\right)} \\
& \geq \frac{\pi}{\sqrt{k} \log R}=a .
\end{aligned}
$$

This implies that $\rho_{a}(z)|d z| \in \Gamma(a)$ since any path in $\Omega(r)$ having index \pm 1 with respect to the origin has length at least 1 relative to $\sigma_{\Omega(r)}(z)|d z|$. Direct calculation gives

$$
A_{\rho_{a}}(\Omega(r))=(1 / k) A_{\lambda_{R}}(\Omega(r))=\frac{2 \pi^{2}}{k \log R} \tan \left(\frac{\pi \log r}{2 \log R}\right),
$$

so that

$$
\begin{aligned}
\bmod (\Omega(r)) & =\frac{1}{2 \pi} \log r^{2} \\
& =\frac{2 \log R}{\pi^{2}} \arctan \left(\frac{k \log R}{2 \pi^{2}} A_{\rho_{a}}(\Omega(r))\right)=\Psi_{k}\left(A_{\rho_{a}}(\Omega(r))\right) .
\end{aligned}
$$

Thus, $\rho_{a}(z)|d z|$ is an extremal metric.

The preceding work also implies that the extremal metric $\rho_{a}(z)|d z|$ for any $k \geq 0$ has an extremal area property analogous to that of $\sigma_{a}(z)|d z|$ mentioned in §3. If $\rho(z)|d z| \in \Gamma(a)$ and $\kappa(z, \rho) \leq-k$, then we have

$$
\Psi_{k}\left(A_{\rho_{a}}(X)\right)=\bmod (X) \leq \Psi_{k}\left(A_{\rho}(X)\right) .
$$

Because $\Psi_{k}$ is increasing, we obtain

$$
A_{\rho_{a}}(X) \leq A_{\rho}(X) .
$$

A careful analysis of the proof of Theorem 1 in the special case $\Phi=\Phi_{k}$ reveals that $\rho_{a}(z)|d z|$ is the unique extremal metric, so we actually have strict inequality in (10) unless $\rho(z)|d z|=\rho_{a}(z)|d z|$. This is an exact analog of the result for $\sigma_{a}(z)|d z|$.

6. Open question. At the end of section 5 we tried to stress the analogy between the extremal metrics $\sigma_{a}(z)|d z|$ and $\rho_{a}(z)|d z|$. Precisely, let $X$ be a proper doubly connected Riemann surface. If $\sigma(z)|d z|$ is a 
metric on $X$ and $\int_{\gamma} \sigma(z)|d z| \geq a$ for any generator $\gamma$ of the fundamental group of $X$, then $A_{\sigma_{a}}(X) \leq A_{\sigma}(X)$ with equality only if $\sigma(z)|d z|=$ $\sigma_{a}(z)|d z|$. On the other hand, if $\rho(z)|d z|$ is a metric on $X$ with $\kappa(z, \rho) \leq$ $-k \leq 0$ and

$$
\log a \leq \int_{\gamma} \log \left[\frac{\rho(z)}{\sigma_{X}(z)}\right] \sigma_{X}(z)|d z|
$$

for any generator $\gamma$ of the fundamental group of $X$, then $A_{\rho_{a}}(X) \leq A_{\rho}(X)$ with equality only if $\rho(z)|d z|=\rho_{a}(z)|d z|$. Does this same conclusion follow if we replace inequality (11) by $\int_{\gamma} \rho(z)|d z| \geq a$ for any admissible path $\gamma$ ? Observe that if $\gamma$ has length 1 relative to $\sigma_{X}(z)|d z|$, then inequality (11) implies this inequality. Of course, an affirmative answer to this question would yield an even closer analogy between the extremal metrics $\sigma_{a}(z)|d z|$ and $\rho_{a}(z)|d z|$.

Added in proof. The question raised in section 6 has been answered affirmatively for the wider class of $S K(k)$ metrics by using the length-area method in conjugation with an isoperimetric inequality (D. Minda, The modulus of a doubly connected region and negatively curved metrics, Complex Variables Theory Appl., to appear).

\section{REFERENCES}

[1] L. V. Ahlfors, An extension of Schwarz's lemma, Trans. Amer. Math. Soc., 43 (1938), 359-364.

[2] Geodesic Curvature and Area, Studies in Mathematical Analysis and Related Topics, ed. by Gilbarg, Solomon and others, Stanford Univ. Press, Stanford, Calif., 1962, pp. 1-7.

[3] M. Heins, On a class of conformal metrics, Nagoya Math. J., 21 (1962), 1-60.

[4] J. A. Jenkins, On the existence of certain general extremal metrics, Ann. of Math., 66 (1957), 440-453.

[5] C. D. Minda, A refinement of Ahlfors' lemma, Complex Variables: Theory and Application, 1 (1983), 167-179.

Received November 1, 1982. The author received support under NSF Grant No. MCS8201131 .

UNIVERSITY OF CINCINNATI

Cincinnati, OH 45221 


\section{PACIFIC JOURNAL OF MATHEMATICS \\ EDITORS}

Donald BaBBITT (Managing Editor)

University of California

Los Angeles, CA 90024

Hugo RossI

University of Utah

Salt Lake City, UT 84112

C. C. Moore and Arthur Ogus

University of California

Berkeley, CA 94720
J. DugundiI

Department of Mathematics

University of Southern California

Los Angeles, CA 90089-1113

R. FINN and H. SAMELSON

Stanford University

Stanford, CA 94305

\section{ASSOCIATE EDITORS}
R. ARENS
E. F. BECKENBACH
B. H. NeUMANN
F. WOLF
K. YoshidA (1906-1982)

\section{SUPPORTING INSTITUTIONS}

\author{
UNIVERSITY OF ARIZONA \\ UNIVERSITY OF BRITISH COLUMBIA \\ CALIFORNIA INSTITUTE OF TECHNOLOGY \\ UNIVERSITY OF CALIFORNIA \\ MONTANA STATE UNIVERSITY \\ UNIVERSITY OF NEVADA, RENO \\ NEW MEXICO STATE UNIVERSITY \\ OREGON STATE UNIVERSITY
}

\author{
UNIVERSITY OF OREGON \\ UNIVERSITY OF SOUTHERN CALIFORNIA \\ STANFORD UNIVERSITY \\ UNIVERSITY OF HAWAII \\ UNIVERSITY OF TOKYO \\ UNIVERSITY OF UTAH \\ WASHINGTON STATE UNIVERSITY \\ UNIVERSITY OF WASHINGTON
}

The Supporting Institutions listed above contribute to the cost of publication of this Journal, but they are not owners or publishers and have no responsibility for its content or policies.

Mathematical papers intended for publication in the Pacific Journal of Mathematics should be in typed form or offset-reproduced (not dittoed), double spaced with large margins. Please do not use built up fractions in the text of the manuscript. However, you may use them in the displayed equations. Underline Greek letters in red, German in green, and script in blue. The first paragraph must be capable of being used separately as a synopsis of the entire paper. In particular it should contain no bibliographic references. Please propose a heading for the odd numbered pages of less than 35 characters. Manuscripts, in triplicate, may be sent to any one of the editors. Please classify according to the scheme of Math. Reviews, Index to Vol. 39. Supply name and address of author to whom proofs should be sent. All other communications should be addressed to the managing editor, or Elaine Barth, University of California, Los Angeles, California 90024.

There are page-charges associated with articles appearing in the Pacific Journal of Mathematics. These charges are expected to be paid by the author's University, Government Agency or Company. If the author or authors do not have access to such Institutional support these charges are waived. Single authors will receive 50 free reprints; joint authors will receive a total of 100 free reprints. Additional copies may be obtained at cost in multiples of 50 .

The Pacific Journal of Mathematics is issued monthly as of January 1966. Regular subscription rate: $\$ 132.00$ a year (6 Vol., 12 issues). Special rate: $\$ 66.00$ a year to individual members of supporting institutions.

Subscriptions, orders for numbers issued in the last three calendar years, and changes of address should be sent to Pacific Journal of Mathematics, P.O. Box 969, Carmel Valley, CA 93924, U.S.A. Old back numbers obtainable from Kraus Periodicals Co., Route 100, Millwood, NY 10546.

The Pacific Journal of Mathematics ISSN 0030-8730 is published monthly by the Pacific Journal of Mathematics at P.O. Box 969, Carmel Valley, CA 93924. Application to mail at Second-class postage rates is pending at Carmel Valley, California, and additional mailing offices. Postmaster: Send address changes to Pacific Journal of Mathematics, P. O. Box 969, Carmel Valley, CA 93924.

PUBLISHED BY PACIFIC JOURNAL OF MATHEMATICS, A NON-PROFIT CORPORATION

Copyright $\odot 1984$ by Pacific Journal of Mathematics 


\section{Pacific Journal of Mathematics}

\section{Vol. 113, No. $2 \quad$ April, 1984}

Alan Adolphson, On the Dwork trace formula ...................257

Amos Altshuler and Leon Steinberg, Enumeration of the quasisimplicial

3 -spheres and 4-polytopes with eight vertices .................. 269

Kenneth R. Goodearl, Cancellation of low-rank vector bundles .......... 289

Gary Fred Gruenhage, Ernest A. Michael and Yoshio Tanaka, Spaces

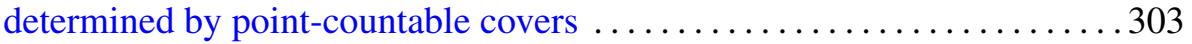

Charles Lemuel Hagopian, Atriodic homogeneous continua .......... 333

David Harbater, Ordinary and supersingular covers in characteristic $p$. . . 349

Domingo Antonio Herrero, Continuity of spectral functions and the lakes

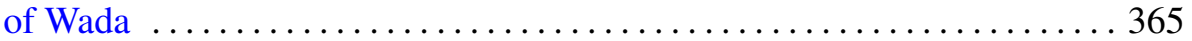

Donald William Kahn, Differentiable approximations to homotopy resolutions and framed cobordism ....................... 373

K. McGovern, On the lifting theory of finite groups of Lie type $\ldots \ldots \ldots . \ldots 383$

C. David (Carl) Minda, The modulus of a doubly connected region and the geodesic curvature-area method ............................. 395

Takuo Miwa, Complexes are spaces with a $\sigma$-almost locally finite base $\ldots . .407$

Ho Kuen Ng, Finitely presented dimension of commutative rings and modules

Roger David Nussbaum, A folk theorem in the spectral theory of

$C_{0}$-semigroups

J. S. Okon, Prime divisors, analytic spread and filtrations

Harold Raymond Parks, Regularity of solutions to elliptic isoperimetric problems

R. Sitaramachandra Rao and M. V. Subba Rao, Transformation formulae for multiple series

Daniel Ruberman, Imbedding punctured lens spaces and connected sums

Uri Srebro, Deficiencies of immersions 